\title{
THE MAGNETIC FIELD IN THE INNER 70 PARSECS OF THE MILKY WAY
}

\author{
MARK MORRIS \\ Department of Astronomy \\ University of California, Los Angeles \\ Los Angeles, CA 90024 \\ USA
}

\begin{abstract}
Existing radiofrequency data on the center of the Galaxy are used to defend the hypothesis that the magnetic field within a radius of at least $70 \mathrm{pc}$ of the nucleus has a poloidal geometry, is relatively uniform in strength, and is very strong relative to extended fields measured elsewhere in the Galaxy, having a flux density greater than or about a milligauss. The implications of this strong, pervasive field are: 1) that a strong ring current is present at radii beyond $70 \mathrm{pc}$, and 2) that the dynamics of molecular clouds in the Galactic center are significantly affected by, though probably not dominated by, the magnetic field. At radii within $5 \mathrm{pc}$, far-IR polarization data suggest a substantial deviation of the magnetic field from a poloidal geometry, at least within the gas and dust layer. There, the coupling of the field to the rapidly orbiting gas near the nucleus is presumably responsible for this local distortion of the larger-scale field geometry.
\end{abstract}

\section{Introduction}

In recent years, increasing attention has been focussed on the magnetic fields of galactic nuclei, as the manifestations of such fields fall within the reach of our observational techniques. The papers of this conference attest to the growth of reliable data bearing on the magnetic fields within the central regions of galaxies. Of course, the proximity of the nucleus of our own Galaxy makes it by far the best sample of a nuclear environment. There, the resolution and sensitivity of observations are at least two orders of magnitude better than can be obtained elsewhere with the same techniques. Consequently, we can ultimately expect to acquire a relatively detailed picture of the Galactic center magnetosphere, and perhaps use it as a model against which to compare other, less clearly viewed nuclear regions. Indeed, high-resolution observations since 1984 of filamentary radio structures in the inner galaxy (Yusef-Zadeh et al. 1984) provide a direct measure of the magnetic field orientation, projected onto the plane of the sky, at several locations (reviewed by YusefZadeh 1989). It is the purpose of this article to point out that a rather clear pattern is emerging from the several filamentary systems that have now been observed: within at least the inner $70 \mathrm{pc}$ of the Galaxy, the magnetic field is remarkably strong and well-ordered, with an overall poloidal geometry. 


\section{The Geometry of the Field}

The argument for the geometry of the magnetic field is based on the existence and the orientation of several filamentary radio structures in the Galactic center region. These are catalogued in Table 1.

TABLE 1. Known filamentary radio continuum structures near the Galactic center

\begin{tabular}{llcrl}
\hline \hline Name & Galactic & Position & Length & References \\
& Longitude & Angle $^{1}$ & (pc) & \\
\hline
\end{tabular}

The Arc:

\begin{tabular}{|c|c|c|c|c|}
\hline - main body & 0.15 to $0.20^{\circ}$ & $0^{\circ}$ & 45 & Yusef-Zadeh et al. 1984, 1986 \\
\hline $\begin{array}{l}\text { - northern } \\
\text { extension }\end{array}$ & 0.15 to $0.20^{\circ}$ & $-10^{\circ}$ to $10^{\circ}$ & 45 & $\begin{array}{l}\text { Yusef-Zadeh and Morris 1987a } \\
\text { Yusef-Zadeh and Morris } 1988\end{array}$ \\
\hline $\begin{array}{l}\text { - southern } \\
\text { extension }\end{array}$ & 0.14 to $0.22^{\circ}$ & $0^{\circ}$ to $40^{\circ}$ & 40 & $\begin{array}{l}\text { Yusef-Zadeh et al. } 1989 \\
\text { (these proceedings) }\end{array}$ \\
\hline $\begin{array}{l}\text { Sgr C } \\
\text { Threads: }\end{array}$ & $359.45^{\circ}$ & $-15^{\circ}$ & 35 & Liszt 1985 \\
\hline $\begin{array}{l}\text { - northern } \\
\text { - southern }\end{array}$ & 0.10 to $0.14^{\circ}$ & $\begin{array}{l}0^{\circ} \text { to } 20^{\circ} \\
-10^{\circ}\end{array}$ & $\begin{array}{l}25 \\
30\end{array}$ & $\begin{array}{l}\text { Morris and Yusef-Zadeh } 1985 \\
\text { Morris and Yusef-Zadeh } 1990\end{array}$ \\
\hline G359.54+0.18 & $359.54^{\circ}$ & $-40^{\circ}$ & 18 & $\begin{array}{l}\text { Bally and Yusef-Zadeh } 1989 \\
\text { Bally et al. } 1989\end{array}$ \\
\hline $\begin{array}{l}\text { G359.79+0.17 } \\
\text { (source K) }\end{array}$ & $359.79^{\circ}$ & $-30^{\circ}$ to $-45^{\circ}$ & 12 & Yusef-Zadeh and Morris 1987b \\
\hline
\end{tabular}

The filaments listed in Table 1 are all within half a degree of the Galactic center - about 70 parsecs, so the comments to follow refer to the magnetic field on this scale. No comparable filaments have been observed outside this region, despite several adequately sensitive observations. A low-frequency (and thus relatively low-resolution) VLA image which simultaneously shows the relative placement and orientation of all of these filament systems is given by Anantharamaiah $e t$ al. in these proceedings.

That the radio filaments are intimately associated with a magnetic field is evidenced by their large percentage polarization, particularly at high frequencies, where the Faraday depolarization is minimal (Inoue et al. 1989). The alignment of the magnetic field with the filaments, suggested by their morphology, was confirmed by Tsuboi et al. (1986), who determined the Faraday rotation, where it could be measured with the resolution of a single dish antenna at $3 \mathrm{~cm}$, in the bundle of filaments comprising the radio Arc. When the electric vectors were corrected for Faraday rotation, and assumed to result from synchrotron emission, they revealed a strong tendency for the magnetic field to follow the filaments. Similar polarization studies of the other filaments are in progress; in the absence of concrete results, we proceed with the assumption that all of the filamentary structures in Table 1 trace the magnetic field direction. 
The field direction thus deduced from the filaments is roughly normal to the Galactic plane at all longitudes, especially as the curvature is followed to the plane. More precisely, the orientation of all of the filaments is consistent with their being part of a poloidal field which diverges somewhat beyond distances of $\sim 25$ parsecs above and below the Galactic plane. The position angle of any given filament is such that, at increasing distance from the Galactic plane, it leans away from the Galaxy's rotation axis. This is well exemplified by the filaments in the region to the south of the radio Arc (Yusef-Zadeh et al., in these proceedings), and by G359.54+0.18 (Bally et al. 1989).

There are other morphological indications that a poloidal field is present in this region, though the other evidence is not as strong as for the filaments. First, there is the Galactic Center Lobe, composed of two 150 -pc tongues of radio emission rising out of the Galactic plane toward positive latitudes, and straddling the Galactic nucleus (Sofue and Handa 1984; Sofue 1985). These features merge continuously with the Arc on one side of the nucleus, and with Sgr C on the other. One might interpret the Galactic Center Lobe, if it is indeed a single, coherent structure (see K. Uchida et al. 1989), as a limb-brightened "chimney" which resulted either from an explosion in the presence of a poloidal magnetic field (Umemura et al. 1988), or from the dynamical twisting of a preexisting poloidal field (Y. Uchida et al. 1985). These MHD models are summarized by Shibata (1989).

Another argument for the presence of a poloidal field was forwarded by Yusef-Zadeh and Morris (1987b) on the basis of the orientation of the elongated, nonthermal shell source surrounding the nucleus, Sgr A East. The major axis of this elliptical structure is parallel to the Galactic plane, whereas any kind of localized energy release into a density-stratified plane-parallel medium would, in the absence of a magnetic field, result in an elongated structure having the orthogonal orientation. This anomaly might be understood if a poloidal magnetic field is present, since the magnetic disturbance would propagate fastest in the direction perpendicular to the field lines.

Finally, several other linear radio features which are perpendicular to the Galactic plane have been noted, although they don't have the same status as the filaments discussed above, and it is not obvious that they trace the magnetic field. These include the "streamers" emanating out toward positive latitudes from Sgr A West (Yusef-Zadeh and Morris 1987b) and a set of ill-defined, quasi-linear features associated with the arched radio filaments at about $1=0.05^{\circ}$ (Morris and Yusef-Zadeh 1989). (The arched radio filaments, part of the radio Arc, are fleecy thermal structures, and their orientation is presumably not related to the magnetic field direction in any simple way.)

\section{The Strength of the Field}

The linearity of the observed filamentary systems indicates that the field within them is strong. It is evident that the interstellar medium near the Galactic center is very inhomogeneous and that the velocity dispersion of clouds there is large, probably larger than the internal velocity dispersion within individual clouds, $\sim 25 \mathrm{~km} \mathrm{~s}^{-1}$. And yet the filaments are almost all characterized by only a very gentle curvature. A good example is provided by the radio threads, one of which is shown in figure 1 . None of the filaments 


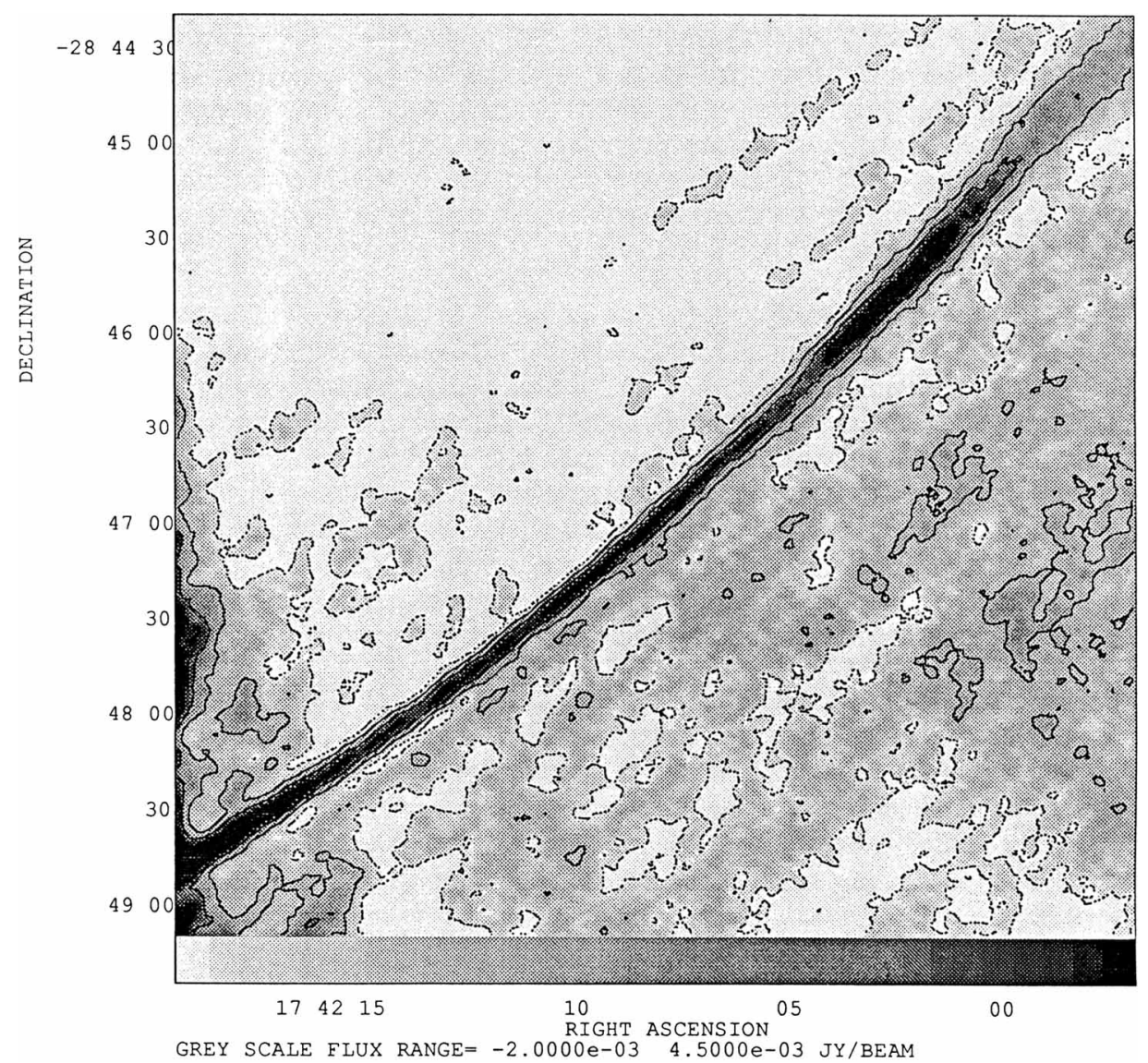

Figure 1: VLA image of the northern radio thread, made at a wavelength of $20 \mathrm{~cm}$. Data from Morris and Yusef-Zadeh (1989, in preparation). The resolution is about 4 arcsec.

suffers any gross contortion such as might be expected to result from the interaction of a portion of a filament with a density inhomogeneity. However, the lengths of the filaments (Table 1) are almost certainly larger than the scale size of density inhomogeneities, as judged from molecular line maps, so such interactions are inevitable. One must conclude that the rigidity of the field in the filaments is large enough to withstand external ram pressures. Even for density fluctuations of a few hundred $\mathrm{cm}^{-3}$, corresponding to minimal molecular clouds, fields on the order of a milligauss are needed.

This kind of argument was used by Yusef-Zadeh and Morris (1987a, 1987c, and 1988) to infer that the filaments of the Arc have at least milligauss field strength. They identify at least three locations at which a molecular cloud is interacting directly with the Arc, and yet the observed distortion of the relevant filaments is only 5 or $10^{\circ}$ at most. In these proceedings, Tsuboi et al. (1989) illustrate one of the clearer cases of a small distortion in a radio filament provoked by the interaction with a molecular cloud . 


\section{The Uniformity of the Field}

Until now, we have not considered whether the field consists of a set of isolated flux tubes, whether it is uniform throughout the inner 70 parsecs, or whether the filaments all lie on a cylindrical magnetic wall surrounding an interior cavity with a negligible field throughout most of its volume (e.g., Heyvaerts et al. 1988). The filaments must be confined by some external pressure; otherwise, their strong fields would cause them to literally explode, and they could not survive for the time that it would take an Alfvén wave to travel their length, i.e., the minimum time that it would take for them to come into being. The external pressure needed to confine them is $\mathrm{n}\left(\mathrm{cm}^{-3}\right) \mathrm{T}(\mathrm{K}) \approx 3 \times 10^{8}$, where $\mathrm{n}$ is the number density of gas particles and $\mathrm{T}$ is the gas kinetic temperature.

The presence of a diffuse $\mathrm{x}$-ray source in the inner galaxy leads one to consider plasma confinement, but the $\mathrm{x}$-rays imply only that $\mathrm{nT} \sim 10^{7} \mathrm{~cm}^{-3} \mathrm{~K}$, if they're produced by Bremsstrahlung (Watson et al. 1981). Furthermore, the extent of the diffuse $\mathrm{x}$-ray source is smaller than the full extent of the Galactic center filaments. We conclude that gas pressure is inadequate to confine the filaments, and that the filaments are not isolated flux tubes. Rather, they are "illuminated" flux tubes within a relatively uniform field. As the illumination appears to be synchrotron emission, these flux tubes are apparently those into which relativistic particles have been injected by a mechanism which remains to be identified.

The idea that a magnetic wall surrounds the nucleus and that the filaments are all embedded in it cannot be ruled out, although the implied system of counter-rotating currents seems rather unlikely. One might speculate that a wall is created as radial motions of matter ejected from the nucleus push back the field and concentrate it at some radius. However, the confinement problem remains; the radial wind would have to be fairly steady to keep the field from expanding inwards, and there is no evidence for a steady wind on a scale of $70 \mathrm{pc}$. We therefore rest with the conclusion that the field strength is uniform within $70 \mathrm{pc}$.

\section{Interactions of Molecular Clouds with the Field}

How does the magnetic field scale with density in this environment? The magnetic field strength is not likely to be strongly concentrated within molecular clouds, as it is elsewhere in the Galactic plane. The field estimated within the clouds is comparable to the external field, by virtue of the fact that we adopt the internal velocity dispersion in molecular clouds to derive a lower limit on the ram pressure to which the filaments are subjected. Indeed, the Alfvén velocity in clouds, assuming a milligauss field and a typical density of $10^{4} \mathrm{~cm}^{-3}$, is about $15 \mathrm{~km} \mathrm{~s}^{-1}$, which is the same as the observed internal velocity dispersion. The ratio of magnetic to gravitational energy within a homogeneous, spherical cloud of radius $\mathrm{R}_{5}$ (in units of $5 \mathrm{pc}$ ) and density $\mathrm{n}_{4}$ (in units of $10^{4} \mathrm{~cm}^{-3}$ ) is $\zeta=0.9\left(\mathrm{~B}_{\mathrm{mG}} / \mathrm{R}_{5} \mathrm{n}_{4}\right)^{2}$. Since, for a typical cloud, $R_{5} \approx n_{4} \approx 1$ (see Gusten 1989), the magnetic field within clouds is indeed unlikely to be much larger than a milligauss. If $\zeta>1$, magnetic pressure would cause the cloud to expand.

Morris (1989) has argued that clouds in the Galactic center are magnetically supported, and that the uniformity of the field direction inhibits cloud collapse to form stars, although he left 
open the possibility that stars could form as the magnetic flux declined by ambipolar diffusion. The support of the cloud along the field direction is presumably provided by Alfvén waves, as described by Shu et al. (1987). The comparability of field strengths external and internal to clouds may mean that the efficiency of ambipolar diffusion is reduced relative to regions where the external field is negligible. So the slow formation of stars by ambipolar diffusion of the field would indeed be inhibited in this environment.

Clouds moving through the field will experience a drag force caused by the field. The magnetic drag can be estimated by noting that, in front of a cloud moving with velocity $\mathrm{V}$ with respect to the field lines, the field will be compressed by the factor $\left(1+V / V_{A}\right)$, where $V_{A}=B /\left(4 \pi \rho_{i c m}\right)^{1 / 2}$ is the Alfvén velocity in the intercloud medium in which the density is $\rho_{\text {icm. }}$. Then the pressure difference between the front and the back of the cloud is

$$
\left(4 \mathrm{~V} / \mathrm{V}_{\mathrm{A}}\right) \mathrm{B}^{2} / 8 \pi=\mathrm{BV}\left(\rho_{\mathrm{icm}} / \pi\right)^{1 / 2} \text {. }
$$

The resulting deceleration of a spherical cloud of radius $r$ and density $\rho$ is

$$
\mathrm{dV} / \mathrm{dt}=3 \mathrm{BV}\left(\rho_{\mathrm{icm}} / \pi\right)^{1 / 2} /(4 \mathrm{R} \rho),
$$

and the time scale for deceleration of the cloud is therefore

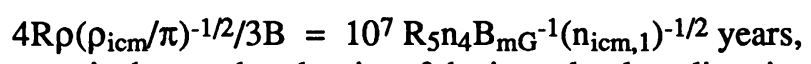

where $n_{\mathrm{icm}, 1}$ is the number density of the intercloud medium in units of $10 \mathrm{~cm}^{-3}$. We conclude that the events giving rise to the observed non-circular motions of clouds in the Galactic center must occur on a time scale at least as short as $\sim 10^{7}$ years.

\section{Implied Current}

The lack of distortion of the poloidal field as it passes through the Galactic plane implies that the field is not anchored in the gas layer. It is perhaps coupled to the Galactic halo gas (Yusef-Zadeh and Morris 1987a). In any case, the confinement of the field to the central 70 pc must be effected by an enormous ring current at radii of $70 \mathrm{pc}$ or beyond. The origin of this current is not addressed here in detail; it is perhaps driven by radial motions (e.g., Lesch et al. 1989). In this regard, it is interesting that the tremendous kinetic energy of the expanding molecular ring (5 $\times 10^{54}$ ergs; Sanders 1989) is comparable to the total magnetic energy in the inner $70 \mathrm{pc}\left(1-2 \times 10^{54} \mathrm{~B}(\mathrm{mG})^{2}\right.$ ergs $)$. One might also speculate that the current is somehow driven by the inexorable inward migration of matter toward the Galactic center (Sofue 1989), in which case the energy source is the release of gravitational potential energy.

\section{The Inner 5 Parsecs}

Far-infrared polarization observations of the dust in the circumnuclear disk at radii of $\sim 5 \mathrm{pc}$, and $10 \mu \mathrm{m}$ observations of the northern arm of Sgr A West, located within the disk, show a predominant polarization angle, which, when ascribed to magnetically aligned dust grains, implies that the magnetic field in the inner region of the Galactic center magnetosphere is predominantly parallel to the Galactic plane (Werner et al. 1988; Aitken 1989 and references therein). These measurements refer to the dust in relatively dense structures, and indicate that the field strength may rise to $10 \mathrm{mG}$ there. The lack of correspondence between the larger-scale poloidal field and the field in the dense interior clouds might be interpreted to mean that the clouds are not magnetically coupled to the surrounding volume. It is 
reasonable to expect that the differential rotation of the circumnuclear disk, coupled with flux-freezing of the field to the partially ionized medium of the disk (Genzel 1989), pulls the field within the dense medium into a predominantly toroidal configuration. The detailed polarization observations show, however, that the field is more complex than one which is purely toroidal (Hildebrand et al. 1990). The use of far-infrared polarimetry to study the magnetic field in this region is just beginning. This technique should ultimately provide quite a detailed picture of the field geometry in and around the circumnuclear disk.

This work was supported by NSF grant AST87-18068 to UCLA.

\section{REFERENCES}

Aitken, D.K. (1989) in M. Morris (ed.), The Center of the Galaxy, Kluwer Acad., Dordrecht, pp. 457 - 464.

Bally, J. and Yusef-Zadeh, F. (1989) Ap.J., 336, 173.

Bally, J., Yusef-Zadeh, F. and Hollis, J.M. (1989) in M. Morris (ed.), The Center of the Galaxy, Kluwer Academic Publishers, Dordrecht, pp. 189-194.

Genzel, R. (1989) in M. Morris (ed.), The Center of the Galaxy, Kluwer Acad., Dordrecht, pp. 393 - 406.

Gusten, R. (1989) in M. Morris (ed.), The Center of the Galaxy, Kluwer Acad., Dordrecht, pp. 89 - 106.

Heyvaerts, J., Norman, C. and Pudritz, R.E. (1988) Ap.J., 330, 718.

Hildebrand, R.H., Novak, G., Platt, S.R., Gonatos, D., Wu, X., Davidson, J. and Werner, M.W. (1990) in preparation.

Inoue, M., Fomalont, E., Tsuboi, M., Yusef-Zadeh, F., Morris, M., Tabara, H. and Kato, T. (1989) in M. Morris (ed.), The Center of the Galaxy, Kluwer Academic Publ., Dordrecht, pp. 269-275.

Lesch, H., Crusius, A., Schlickeiser, R. and Wielebinski, R. (1989) in these proceedings.

Liszt, H.S. (1985) ApJ. (Letters), 239, L65.

Morris, M. (1989) in M. Morris (ed.), The Center of the Galaxy, Kluwer Acad., Dordrecht, pp. 171 - 177.

Morris, M. and Yusef-Zadeh, F. (1985), Astron. J., 90, 2511.

Morris, M. and Yusef-Zadeh, F. (1989) Ap.J., 343, pp. 703 - 712.

Sanders, R. (1989) in M. Morris (ed.), The Center of the Galaxy, Kluwer Acad., Dordrecht, pp. 77 - 87.

Shibata, K. (1989) in M. Morris (ed.), The Center of the Galaxy, Kluwer Acad., Dordrecht, pp. 313-317.

Shu, F.H., Adams, F.C. and Lizano, S. (1987) Ann. Rev. Astron. Astrophys., 25, 23.

Sofue, Y. (1985) Pub. Astr. Soc. Japan, 37, 697.

Sofue, Y. (1989) in these proceedings.

Sofue, Y. and Handa, T. (1984) Nature, 310, 568.

Tsuboi, M., Inoue, M., Handa, T., Inatani, J. and Ukita (1989) in these proceedings.

Tsuboi, M., Inoue, M., Handa, T., Tabara, H., Kato, T., Sofue, Y. and Kaifu, N. (1986) Astron. J., 92, 818.

Uchida, K., Morris, M. and Serabyn, E. (1989) Ap.J., in press.

Uchida, Y., Shibata, K. and Sofue, Y. (1985) Nature, 317, 699.

Umemura, S., Iki, K., Shibata, K. and Sofue, Y. (1988) Pub. Astr. Soc. Japan., 40, 25.

Watson, M.G., Willingale, R., Grindlay, J.E. and Hertz, P. (1981), Ap.J., 250, 142.

Werner, M.W., Davidson, J.A., Morris, M., Novak, G., Platt, S.R., and Hildebrand, R.H. (1988) Ap.J., 333, 729.

Yusef-Zadeh, F. (1989) in M. Morris (ed.), The Center of the Galaxy, Kluwer Acad., Dordrecht, pp. 243-263.

Yusef-Zadeh, F. and Morris, M. (1987a) Ap.J., 322, 721.

(1987b) Ap.J., 320, 545.

(1987c) Astron. J., 94, 1128.

(1988) Ap.J., 329, 729.

Yusef-Zadeh, F., Morris, M. and Chance, D. (1984) Nature, 310, 557.

Yusef-Zadeh, F., Morris, M., Lasenby, A., Seiradakis, J. and Wielebinski, R. (1989) in these proceedings.

Yusef-Zadeh, F., Morris, M., Slee, O.B., and Nelson, G.J. (1986) Ap.J., 310, 689. 
PERATT: A number of years ago I had the opportunity to simulate Alfvén's ring current model in galaxies (actually the azimuthal current component of a helical current distribution) and found filamentation and poloidal magnetic fields of the order of a milligauss (A. Peratt, 1984, Sky and Telesc. 68, 118-122; H. Alfvén, 1985, Science 231, 909). In fact, the simulations suggested field strengths of $10 \mathrm{mG}$ at the Galactic center. Is this consistent with your discovery?

MORRIS: Ten milligauss may be found in the inner $5 \mathrm{pc}$ or so, but the geometry there appears toroidal. Further out, $10 \mathrm{mG}$ would be perhaps too strong, as the field would then dominate the dynamics of clouds.

DEINZER: What are your comments on the origin of the magnetic fields? Could it not be dynamo-produced in the ring?

MORRIS: The circumnuclear ring at 3 to $10 \mathrm{pc}$ is too small to be the locale of the responsible dynamo. However, the 170 pc expanding molecular ring (EMR) may have been responsible for the generation of the field when it was smaller. Its current kinetic energy $\left(\sim 10^{54}\right.$ ergs $)$ is comparable to the energy in the $50 \mathrm{pc}$ magnetosphere, so allowing for its deceleration in the last $10^{6} \mathrm{yrs}$, the energy of the EMR could have been tapped via a dynamo process to produce the field. The mechanism has yet to be identified, but see the contribution by Lesch et al. (this volume).

One must also consider the hypothesis by Sofue, according to which the vertical component of the primordial field has been concentrated over time in the Galactic center region.

UCHIDA: When you say that there is no evidence of twisting you mean small-scale one or filament twist. There is evidence that the line-ofsight magnetic field component reverses sign on the north and south of Galactic center lobes (Inoue and Tsuboi) indicating that there is a largescale twisting in which the field is pulled by the rotation of the cloud around the Galactic center.

MORRIS: This rotation measure reversal is along the line of sight. The straightness of filaments makes me feel that there is no twist in the field responsible for the filament.

LOW: I like to know if there is firm observational evidence for taking the filaments near the Galactic center to be stationary structures rather than moving objects or fronts.

MORRIS: Being synchrotron-emitting, nonthermal structures, they have no known spectral features with which to measure their velocity. We have observed them twice and have ruled out only velocities near the speed of light using the limit on proper motion.

DURIC: With regard to the strength of the Galactic magnetic field in the inner $50 \mathrm{pc}$, I would like to point out that equipartition calculations for some external galaxies indicate values of a few times $\mathrm{x} 0.1 \mathrm{mG}$. These values are based on high resolution VLA images of nearby, relatively normal spiral galaxies for the inner $50 \mathrm{pc}$. The values found are consistent with those given by $M$. Morris for the filaments in the inner 50 pc of our Galaxy. 